\title{
Cranberry for Bacteriuria in Individuals with Spinal Cord Injury: A Systematic Review and Meta-Analysis
}

\author{
Anna Raguzzini, ${ }^{1}$ Elisabetta Toti, ${ }^{1}$ Tommaso Sciarra $\mathbb{D}^{2},{ }^{2}$ Anna Lucia Fedullo, ${ }^{1}$ \\ and Ilaria Peluso ${ }^{1}{ }^{1}$ \\ ${ }^{1}$ Research Centre for Food and Nutrition, Council for Agricultural Research and Economics (CREA-AN), Rome, Italy \\ ${ }^{2}$ Joint Veteran Center, Scientific Department, Army Medical Center, Rome, Italy \\ Correspondence should be addressed to Tommaso Sciarra; sciarratommaso@hotmail.com and Ilaria Peluso; i.peluso@tiscali.it
}

Received 11 June 2020; Revised 6 August 2020; Accepted 12 October 2020; Published 31 October 2020

Academic Editor: Luciano Saso

Copyright ( 2020 Anna Raguzzini et al. This is an open access article distributed under the Creative Commons Attribution License, which permits unrestricted use, distribution, and reproduction in any medium, provided the original work is properly cited.

\begin{abstract}
Background. Urinary tract infection (UTI) is common in individuals with spinal cord injury (SCI) and neurogenic lower urinary tract dysfunction (NLUTD) and in veterans with SCI who use antibiotics improperly for asymptomatic bacteriuria. Cranberry (CB) has been suggested for UTI prevention. Methods. We performed a systematic search up to May 2020 in the following databases: AccessMedicine, BioMed Central, CINAHL, Cochrane Library, ProQuest, and PubMed. Quality assessment was performed using a specifically designed quality score. Risk ratio was calculated with both random effect model analysis (DerSimonian-Laird method) and quality effect model analysis (Doi Thalib method). Results. Six studies on bacteriuria and SCI were reviewed. From the four studies available for meta-analysis, two of which with individuals taking both CB and control, 477 data from 415 participants were analysed (241 CB and 236 control). No significant differences were detected with meta-analysis. However, bias, limitations, and incompleteness were observed in the reviewed studies. Conclusion. Although further studies are needed, we suggest an accurate monitoring of diet and fluid intake, the evaluation of risk for potential food or nutraceutical interactions with drugs, and the inclusion of inflammatory markers among the outcomes in addition to UTI.
\end{abstract}

\section{Introduction}

Spinal cord injury (SCI) is a damage to the spinal cord that may result in motor paralysis and sensory loss below the level of the lesion [1]. The highest documented global prevalence of SCI was found in the United States of America (906/million) and the lowest in France (250/million), while the incidence of traumatic SCI was highest in New Zealand (49.1/million) and lowest in Spain (8.0/million) [2]. In Italy, the incidence of traumatic SCI during 2013-2014 was $14.7 /$ million per year, the mean age was 54 years old, and the male to female ratio was $4: 1$ [3]. High incidence was documented in veterans. In a systematic review of 25 articles, the overall incidence rate of war-related SCI varied from 4.3 to 5.6/10,000 person-years [4]. These veterans had predominantly thoracic or lumbar level, complete (American Spinal Injury Association [ASIA] Impairment Scale A) SCI, associated with other bodily injuries in $43.9-78.1 \%$ of cases [4].
Moreover, polypharmacy is common in veterans $[5,6]$, and nutraceutical-drug [5] and food-drug interactions [6] should be considered.

Neurogenic lower urinary tract dysfunction (NLUTD) can be observed in $83 \%$ of veterans with SCI and is associated with urinary tract infection (UTI) [7]. Results of routine testing reported that $69 \%$ of the urine cultures of veterans with SCI were positive for bacteria, but $87 \%$ were asymptomatic bacteriuria cases, of which $36 \%$ were treated with antibiotics [8]. Epidemiology of antibiotic resistance in veterans with SCI suggests improving prescribing of appropriate antibiotics [9]. There is a large consensus that antibiotic prophylaxis is not recommended for UTI [10-12] and that asymptomatic bacteriuria should not be treated with antibiotics [13].

Although cranberry (CB) may be effective in preventing UTI recurrence in women [14], data from meta-analyses have reported conflicting results, and it has been suggested that conclusions on cranberry and UTI should consider the 
differences among the populations studied [15]. Sappal et al. [16] recently investigated the effect of concentrated proanthocyanidins (PAC) from CB for reduction of bacteriuria in male veterans with SCI and did not find reduction of bacteriuria and pyuria or improvement in subjective urine quality.

1.1. Aim and Objectives. We aimed to evaluate the following hypothesis: $\mathrm{CB}$ products (including extracts) are more effective than placebo or no treatment in reducing bacteriuria and/or in the prevention of UTI in individuals with SCI. Furthermore, we aimed to suggest a specific quality assessment for studies with nutraceuticals involving individuals with SCI.

To this aim, we conducted a systematic review and evaluated previous meta-analysis risk of bias assessment [1719]. Although it was not a meta-analysis, we also considered the systematic review of Navarrete-Opazo et al. [20], because it was focused on individuals with SCI.

\section{Materials and Methods}

2.1. Study Selection. Figure 1 shows the four-phase diagram of meta-analysis (according to the PRISMA Statement) and the flow of the studies processed in this review. We performed a systematic search in PubMed and in the Discovery Sapienza/medicine pharmacy and psychology including (among others) the following databases: AccessMedicine, BioMed Central, CINAHL, Cochrane Library, and ProQuest, with the search terms spinal cord injury and cranberry, up to May 2020 (Figure 1).

Given that the aim of the present review was specifically SCI, interventions that involved individuals with other health conditions were excluded. In particular, we excluded studies involving subjects with spina bifida, who had higher urinary tumor growth factor $\beta-1$ than patients with SCI [21], and children with myelomeningocele [22], being children among the groups where $\mathrm{CB}$ products seemed to be more effective (relative risk: RR range -0.33 [18] -0.48 [17]). Moreover, these are two congenital conditions [23], and this review is aimed at evaluating the effect of CB in individuals with traumatic SCI.

All studies that met the following criteria were included in this review: studies that appear in an edited journal (peer-review criterion), published in English (language criterion), and focused on the effect of CB on bacteriuria or UTI versus control (topic criterion), regardless of the $\mathrm{CB}$ bioactive compounds' source and dose and the study design (parallel, crossover, controlled, and uncontrolled). First trials were identified through the title or abstract. Then, the full text of the article was obtained. Finally, based on inclusion and exclusion criteria, eligible studies were included (A.R. and E.T.).

2.2. Data Extraction and Quality Assessment. A data extraction form, including quality characteristics, was designed, and selected studies were reviewed by all authors. To ensure uniformity, data extraction was performed independently by two reviewers (A.R. and E.T.), and all data were entered by these reviewers. Discrepancies were resolved by discussion between the two reviewers, and unresolved disagreement was referred to a third reviewer (I.P.).

Table 1 shows previously reported judgments of studies (Table 1). The previously used (Table 1) assessment of bias tool includes random sequence generation (selection bias), allocation concealment (selection bias), blinding of participants and personnel (performance bias), blinding of outcome assessment (detection bias), incomplete outcome data (attrition bias), selective reporting (reporting bias), and other biases [17], each scored as "high risk," "low risk," or "unclear." Navarrete-Opazo et al. [20] judged studies as unclear risk for reporting bias, because there was no previous registration of the protocols.

In this study, withdrawal was not considered as a bias, being the compliance of volunteers generally due to factors not imputable to researchers [24], according to the quality score previously used for intervention studies with flavonoids [24]. The score (range 0-1) [24] includes proper control (0.3), compliance assessment (0.1), dietary record (food records or food-frequency questionnaires throughout the study, 0.06), food antioxidant intake in subjects' selection criteria (flavonoid-rich food or antioxidant supplement consumption, $0.05)$, washout and/or run-in period (0.05, washout and/or run-in period for crossover studies only run-in for parallel design), marker of bioavailability (0.05), double blinding (0.05), no funding support (0.03, from profit companies), and no food/supplement donation (0.01).

Intra- and interstudy baseline comparability was removed from the previously suggested score [24] because no mean difference after versus before treatment was calculated, and the corresponding score 0.3 was divided into groups balanced for lesion level/urine collection (0.1), UTI diagnosis after treatment including autonomic dysreflexia (0.1), and comorbidity and drug use specified (0.1).

Discrepancies were resolved through discussions between the two authors who performed the quality assessment (A.R. and T.S.) or through consultation with a third investigator (I.P.).

2.3. Meta-Analysis. Two [16, 25] out of the 6 selected studies $[16,25-29]$ have been excluded from the meta-analysis since results were provided in the form of figures, but we retained them for discussion, as in the previous meta-analysis [24]. With the definition of UTI being different among the studies, we use bacteriuria as the outcome. Moreover, asymptomatic bacteriuria is often improperly treated with antibiotics [8].

Four studies [26-29] met the inclusion criteria and provided data for the analyses of $\mathrm{CB}$ versus control (Figure 1). Dichotomous outcomes (UTI cases in CB and control groups) from each study were collected in order to compute individual-study RR (with 95\% confidence intervals). Random effect model analysis (DerSimonian-Laird method) and quality effect model analysis (Doi Thalib method, by using the quality score as the probability modifier) were reported. Number needed to treat (NNT) was estimated. Statistical heterogeneity was assessed by using the $Q$ statistics for quality effect model and by $t^{2}$ and prediction interval [30] for the random effect model. 


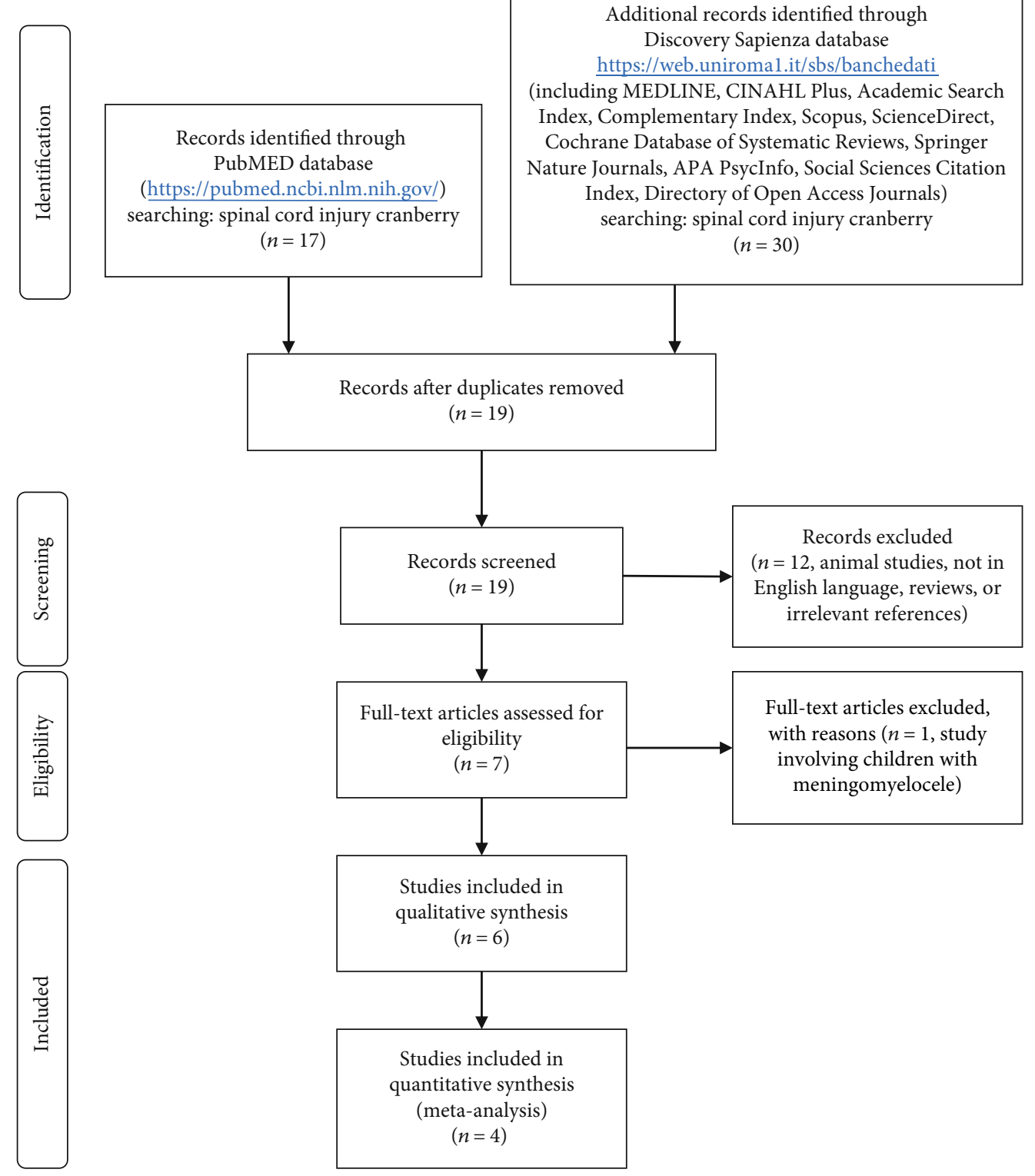

FIGURE 1: Four-phase flow diagram of systematic review and meta-analysis, according to the PRISMA Statement.

In order to detect the presence of publication bias, funnel plot and Egger's weighted regression statistics were used. Symmetry/asymmetry of the funnel plot was defined through visual examination, and trim-and-fill sensitivity analysis was performed. Furthermore, we used the L'Abbe plot [31] in order to visualize the relationship between the effect of treatment and the bacteriuria.

\section{Results}

3.1. Description of Included Studies. Six studies were retrieved from the systematic search, of which 2 crossover, 3 parallel (two 2 arms and one 4 arms), and 1 longitudinal (water before washout and CB juice), with a total of 449 volunteers.

Characteristics of participants, including and excluding selection criteria, are shown in Table 2. Lesion level and ASIA classification were very variable among studies and, when reported, among groups within the same study (Table 2). Reported reasons for dropout/loss of follow-up included noncompliance with pill counts [29], developing of urinary stones [29], recurrent UTI [25], abdominal discomfort attributed to CB [25], and personal reasons, including travels [25].

Only 1 study evaluated the effect of CB juice, whereas the others evaluated CB tablets or capsules (Table 3). Treatment range between 1 week and 6 months and 2 out of 3 crossover studies included a washout period between the two periods of intervention (treatment and control), whereas the other studies did not include a run-in period. The 4-arm study also included a methenamine hippurate $(\mathrm{MH})$ group and a $\mathrm{CB}$ $+\mathrm{MH}$ group. All except for 1 study did not report PAC content in the $\mathrm{CB}$ product. Dropout rates range from $0 \%$ to $43 \%$ 
TABLE 1: Risk of bias from previous meta-analyses.

\begin{tabular}{|c|c|c|c|c|}
\hline $\begin{array}{l}\text { Study name } \\
\text { Year }\end{array}$ & $\begin{array}{c}\text { Linsenmeyer } \\
2004\end{array}$ & $\begin{array}{l}\text { Waites } \\
2004\end{array}$ & $\begin{array}{l}\text { Lee } \\
2007\end{array}$ & $\begin{array}{l}\text { Hess } \\
2008\end{array}$ \\
\hline & \multicolumn{4}{|c|}{ Random sequence generation } \\
\hline Jepson et al. 2012 [17] & Unclear & Unclear & Low & Unclear \\
\hline Wang et al. 2012 [18] & Not reported & Unclear & Not reported & Unclear \\
\hline \multirow[t]{2}{*}{ Luis et al. 2017 [19] } & Not reported & Unclear & Low & Unclear \\
\hline & \multicolumn{4}{|c|}{ Allocation concealment } \\
\hline Jepson et al. 2012 [17] & Unclear & Unclear & Low & Low \\
\hline Wang et al. 2012 [18] & Not reported & Unclear & Not reported & Unclear \\
\hline \multirow[t]{2}{*}{ Luis et al. 2017} & Not reported & Low & Low & Unclear \\
\hline & \multicolumn{4}{|c|}{ Blinding of participants and personnel } \\
\hline Jepson et al. 2012 [17] & Low & Low & Low & Low \\
\hline Wang et al. 2012 [18] & Not reported & Low & Not reported & Low \\
\hline \multirow[t]{2}{*}{ Luis et al. 2017 [19] } & Not reported & Low & Low & Low \\
\hline & \multicolumn{4}{|c|}{ Blinding of outcome assessment } \\
\hline Jepson et al. 2012 [17] & Low & Unclear & Low & Low \\
\hline Wang et al. 2012 [18] & Not reported & Not reported & Not reported & Not reported \\
\hline \multirow[t]{2}{*}{ Luis et al. 2017 [19] } & Not reported & Low & Low & Low \\
\hline & \multicolumn{4}{|c|}{ Incomplete outcome data } \\
\hline Jepson et al. 2012 [17] & Low & High & Low & High \\
\hline Wang et al. 2012 [18] & Not reported & High & Not reported & Unclear \\
\hline \multirow[t]{2}{*}{ Luis et al. 2017 [19] } & Not reported & Low & Unclear & Unclear \\
\hline & \multicolumn{4}{|c|}{ Selective reporting } \\
\hline Jepson et al. 2012 [17] & Low & Low & Low & Low \\
\hline Wang et al. 2012 [18] & Not reported & High & Not reported & High \\
\hline \multirow[t]{2}{*}{ Luis et al. 2017 [19] } & Not reported & Low & Unclear & Low \\
\hline & \multicolumn{4}{|c|}{ Other bias } \\
\hline Jepson et al. 2012 [17] & Unclear & Unclear & Low & Low \\
\hline Wang et al. 2012 [18] & Not reported & Not reported & Not reported & Not reported \\
\hline Luis et al. 2017 [19] & Not reported & Low & Low & Low \\
\hline
\end{tabular}

and different dropout rates were reported for CB (15\%), $\mathrm{CB}+\mathrm{MH}(21 \%)$, placebo (13\%), and $\mathrm{MH}(23 \%)$ in the 4arm study.

The outcomes of studies were symptomatic UTI, bacteriuria plus pyuria (urinary white blood cells count: WBC), or bacteriuria only (Table 3). Among the studies, only Hess et al. [29] reported a significant effect on symptomatic UTI after consumption of CB tablet ( $1 \mathrm{~g} / \mathrm{d}$ for 6 months) compared to placebo. On the other hand, Lee et al. [28] reported bowel dysfunctions (diarrhoea or constipation) in eleven participants, nausea in two, and rash in one, after the treatment with the CB capsule ( $2 \mathrm{~g} / \mathrm{d}$ for 6 months).

3.2. Quality of the Included Studies. Disagreement, between the two authors who performed data extraction and quality assessment, occurred for the study of Lee et al. [28], who did not exclude from the follow-up patients who discontinued the intervention and coupled $\mathrm{CB}$ and $\mathrm{CB}+\mathrm{MH}$ groups and placebo and $\mathrm{MH}$ groups for the analysis. After the consultation with the third reviewer, the study was included in the quantitative synthesis (Figure 1), due to the great number of subjects, but with the lower quality score, according with criteria in Table 4.

The most frequent limitations were no comorbidity and drug use specified (all studies), markers of bioavailability (all studies), and no compliance, diet, or antioxidant monitoring (Table 4).

Only 3 studies included autonomic dysfunction in the symptomatic UTI diagnostic criteria, and in 3 studies, the balancing between treatment and control for the lesion level and urinary collection (management of NLUTD) was not properly controlled.

In particular, Waites et al. [27] stated that groups were unbalanced for catheterization and Lee et al. [28] did not furnish information for the 4 arms of treatment, pooling $\mathrm{CB}$ and $\mathrm{CB}+\mathrm{MH}$ in the treatment group and placebo and $\mathrm{MH}$ in the control group. This kind of analysis is a bias also for proper control (Table 4).

3.3. Meta-Analysis. Since there were 2 studies with individuals taking both treatment and control, 1 crossover [29] and 1 longitudinal [26], 477 data from 415 participants were 


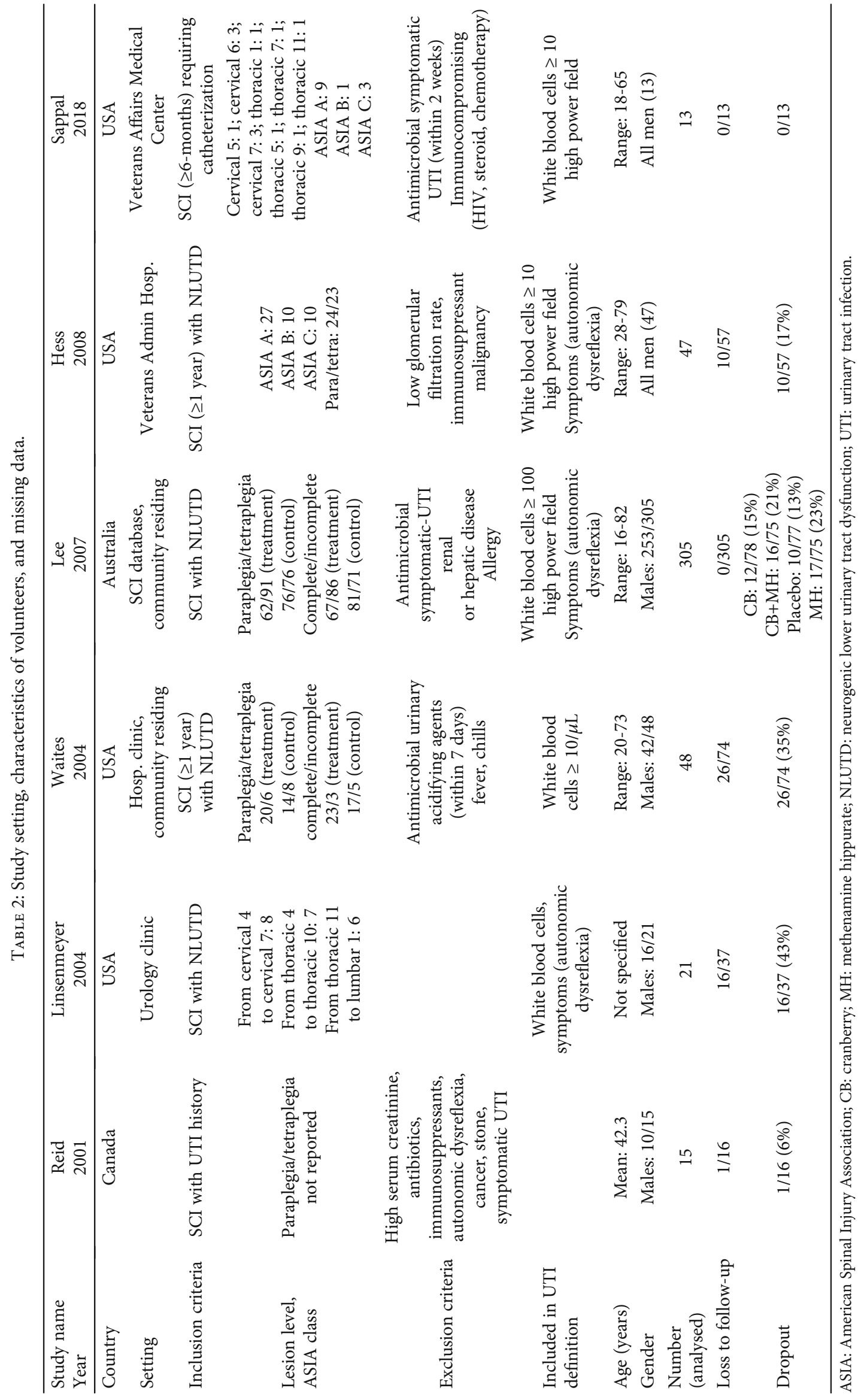




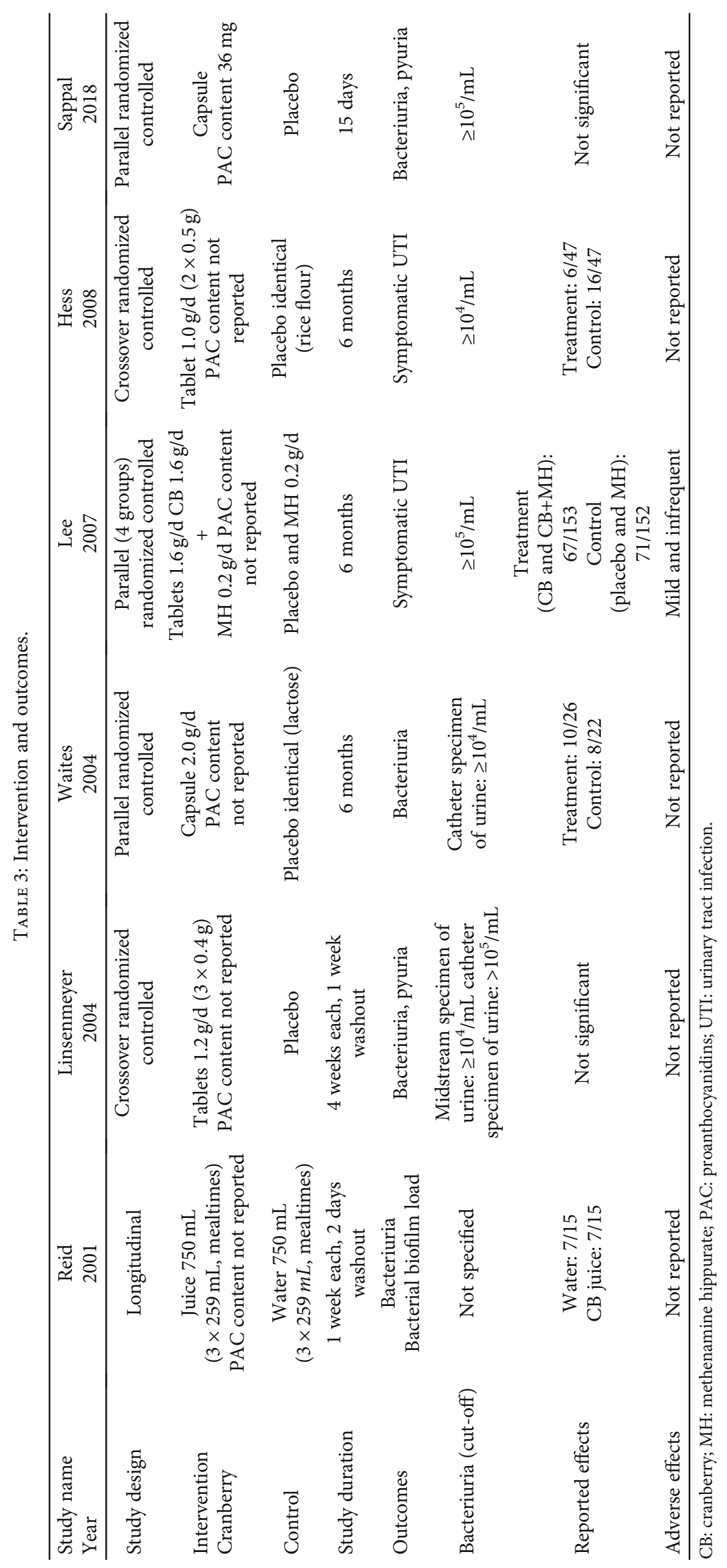


TABLE 4: Specific risk of bias assessment.

\begin{tabular}{|c|c|c|c|c|c|c|}
\hline $\begin{array}{l}\text { Study name } \\
\text { Year }\end{array}$ & $\begin{array}{l}\text { Reid } \\
2001\end{array}$ & $\begin{array}{c}\text { Linsenmeyer } \\
2004\end{array}$ & $\begin{array}{l}\text { Waites } \\
2004\end{array}$ & $\begin{array}{c}\text { Lee } \\
2007\end{array}$ & $\begin{array}{l}\text { Hess } \\
2008\end{array}$ & $\begin{array}{c}\text { Sappal } \\
2018\end{array}$ \\
\hline $\begin{array}{l}\text { Groups balanced for lesion } \\
\text { level/urine collection }\end{array}$ & Yes 0.1 & Yes 0.1 & $\begin{array}{c}\text { Unbalanced catheterization } \\
\text { (treatment/control } 65.4 / 36.3 \% \text { ) }\end{array}$ & $\begin{array}{l}\text { No data for each } \\
\text { of the } 4 \text { arms }\end{array}$ & Yes 0.1 & Not reported \\
\hline $\begin{array}{l}\text { UTI diagnosis including } \\
\text { autonomic dysreflexia }\end{array}$ & No & Yes 0.1 & No & Yes 0.1 & Yes 0.1 & No \\
\hline $\begin{array}{l}\text { Comorbidity and drug use } \\
\text { specified }\end{array}$ & No & No & No & No & No & No \\
\hline Proper control & Yes 0.3 & Yes 0.3 & Yes 0.3 & Placebo $+\mathrm{MH}$ & Yes 0.3 & Yes 0.3 \\
\hline Compliance assessment & No & No & Pills' count 0.1 & No & $\begin{array}{l}\text { Pills' } \\
\text { count } \\
0.1\end{array}$ & No \\
\hline Dietary record & No & No & Only fluid intake 0.03 & No & No & No \\
\hline Food antioxidant intake & $\begin{array}{c}\text { Only no } \\
\text { CB/vit. C } \\
0.025\end{array}$ & $\begin{array}{c}\text { Only no CB } \\
0.025\end{array}$ & Only no CB 0.025 & No & No & Only no CB 0.025 \\
\hline Washout and/or run-in & 2d-w/o only & Yes 0.05 & No & No & No & No \\
\hline Marker of bioavailability & No & No & No & No & No & No \\
\hline Double blinding & No & Yes 0.05 & Yes 0.05 & Yes 0.05 & Yes 0.05 & Yes 0.05 \\
\hline No funding support & $\begin{array}{c}\text { Yes } 0.03 \\
\text { (no conflict) }\end{array}$ & Yes 0.03 & Yes 0.03 & $\begin{array}{c}\text { Brucia } \\
\text { Pharmaceuticals }\end{array}$ & Yes 0.03 & $\begin{array}{c}\text { Yes (critical } \\
\text { versus sponsor) } \\
0.03\end{array}$ \\
\hline No supplement donation & Yes 0.01 & $\begin{array}{c}\text { Kessler } \\
\text { Pharmacy }\end{array}$ & AIM This Way & Yes 0.01 & $\begin{array}{l}\text { Cran- } \\
\text { Max } \\
\text { Swiss }\end{array}$ & Yes 0.01 \\
\hline Quality score (range $0-1$ ) & 0.465 & 0.655 & 0.535 & 0.16 & 0.68 & 0.415 \\
\hline
\end{tabular}

CB: cranberry; MH: methenamine hippurate; UTI: urinary tract infection.

analysed in this meta-analysis. Overall, 241 subjects received $\mathrm{CB}$ and 236 control. No significant differences were detected with meta-analysis in random $(p=0.372)$ and quality $(p=0.415)$ effect models (Figure 2$)$. NNT was 17 (95\% CI 34.36 to 6.82 ), and low to high statistical heterogeneity was found for random and quality effect models, respectively $\left(t^{2}\right.$ 0.05 , Q 75\%, Egger intercept $-0.880, p=0.568)$. However, the $95 \%$ prediction interval ranged between 0.3 and 2.1 . The funnel plot showed no asymmetric distribution of results (Figure 3(a)) and trim-and-fill analysis did not suggest potential publication bias. On the other hand, the visual inspection of the L'Abbe plot revealed that only half of the participants were at risk of UTI in the control group (Figure 3(b)).

\section{Discussion}

4.1. Summary of Main Results. Six studies were retrieved in the present systematic review, 4 of which reported data suitable for the meta-analysis (Figure 1). Considering that inclusion of low-quality studies may bias the estimated effect, while restriction to high-quality studies can reduce information, a quality score has been assigned and a quality effect model meta-analysis has been performed, in addition to the random effect model meta-analysis. We did not observe significant effect of $\mathrm{CB}$ products (Table 3 ) on bacteriuria in individuals with SCI (Table 2 and Figure 2).
About one in every 17 patients will benefit from the treatment. However, NNT does not account for a patient's baseline risk, probably different due to intra- and interstudy variability (Table 2). Moreover, the NNT of a given treatment will be very different when describing the value versus placebo instead of another active therapy (Tables 3 and 4; Lee et al. [28] did not furnish information for the 4 arms of treatment). Moreover, we observed a broad prediction interval (95\% 0.3-2.1), suggesting a range of possible effects in relation to harm and clinical benefit thresholds ( 1 , null effect) and indicating the existence of settings where the treatment has a suboptimal and possibly even harmful effect. None of the studies included in the quantitative synthesis involved the veterans who can be very different from the patients seen in all studies that have been done in the past. Therefore, the prediction interval cannot tell us what we might expect for these patients and specific studies are required to enable more informed clinical decision-making. Moreover, the prediction interval is affected by study bias. If bias exists, as summarized in Table 4, the effect sizes observed in future studies might occur beyond the limits of the prediction interval.

4.2. Completeness, Quality, and Applicability of Evidence. Navarrete-Opazo et al. [20], after a quality assessment across studies, carried out using the Grading of Recommendations Assessment, Development and Evaluation (GRADE), 


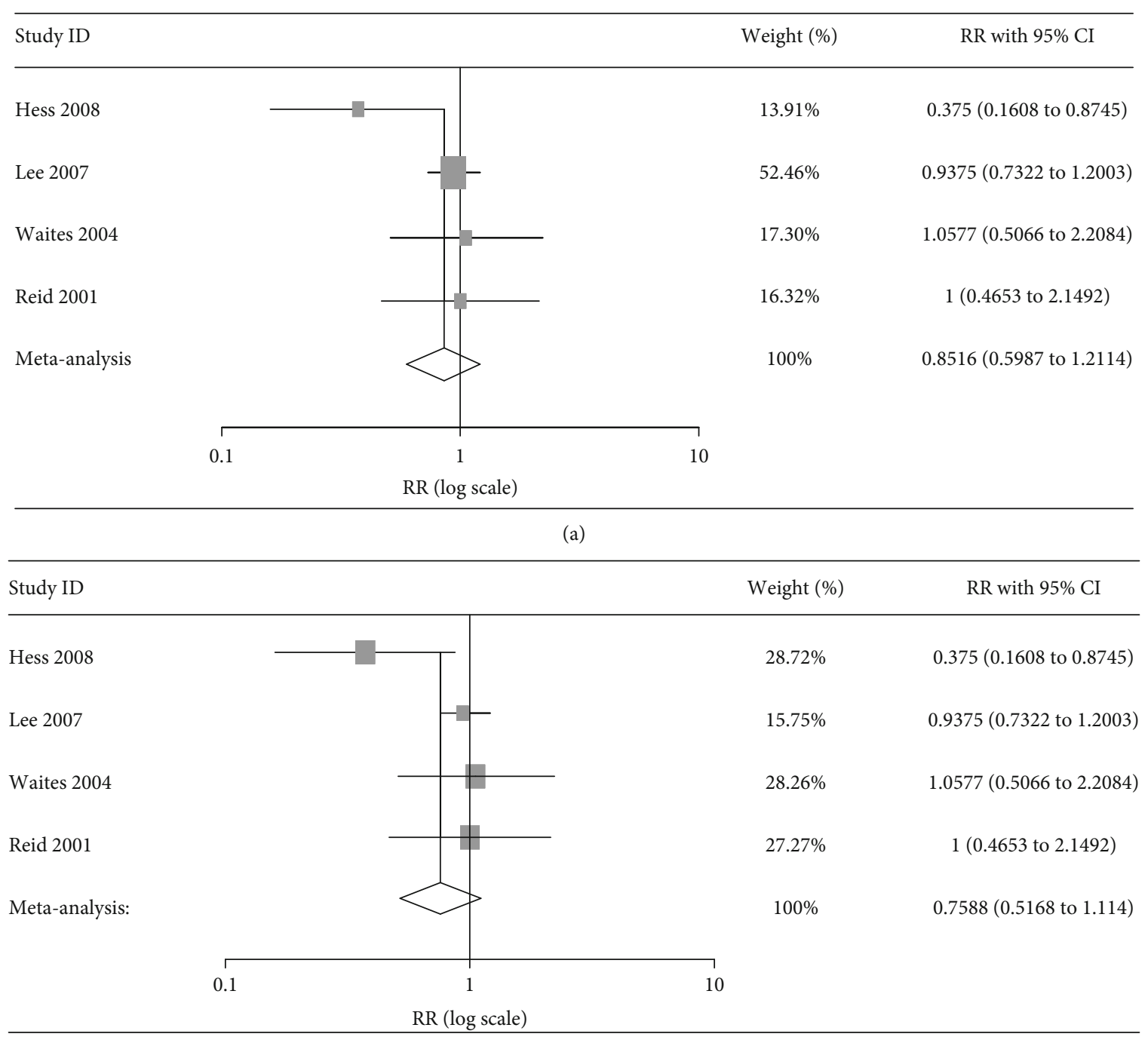

(b)

FIGURE 2: Meta-analysis: (a) forest plot of random effect model; (b) forest plot of quality effect model.

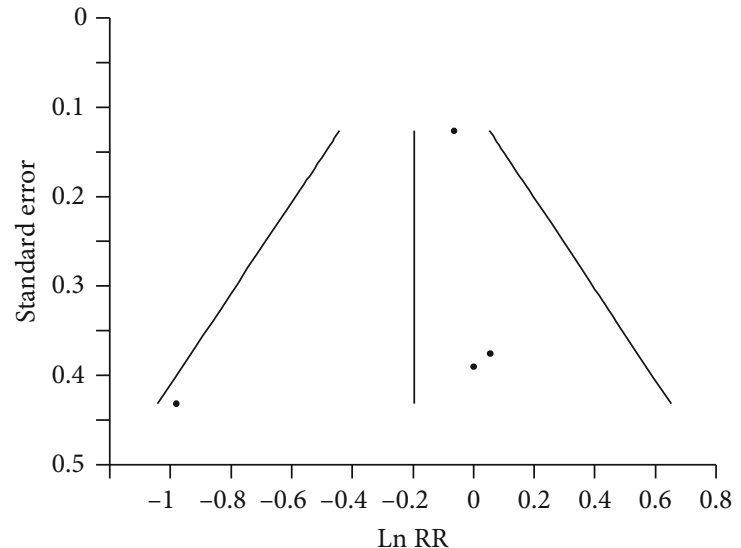

(a)

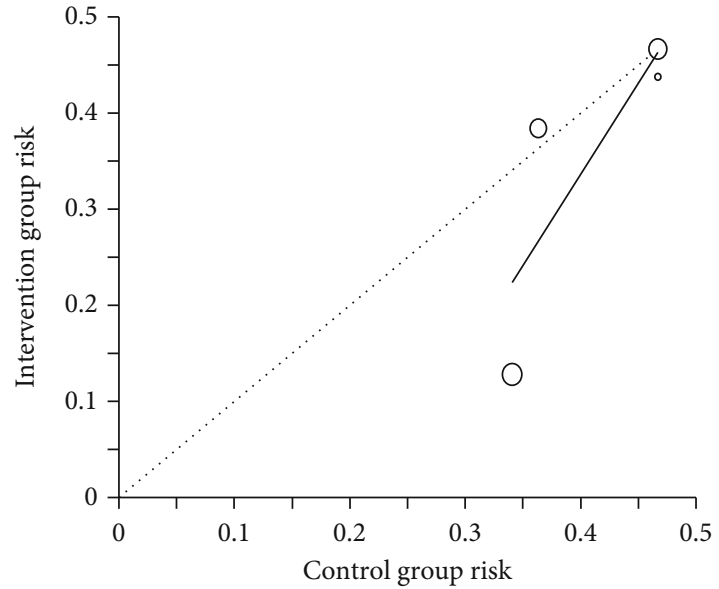

(b)

Figure 3: (a) Funnel plot. Ln RR: risk ratio logarithm. (b) L'Abbe plot. Dotted line: line of equality. 
reported that overall, the studies were rated as moderatequality evidence and the highest quality was found in the trial by Lee et al. [28]. From that, the authors concluded that the quality of the evidence was strong enough to reasonably conclude that cranberry supplementation is not effective for prevention of UTIs in people with SCI [20]. By using our risk of bias assessment (Table 4), we concluded that the quality of evidence is low due to the bias, limitations, and incompleteness of the reviewed studies.

Intersubjects' variability has been reported for absorption, metabolism, and excretion of CB polyphenols in healthy individuals, partly due to the variation in the gut microbiota [32]. Several studies have reported different intestinal microbiota in individuals with SCI compared to healthy controls [33], and diet is known to influence gut microbiota [33] and to affect UTI risk [34]. Therefore, monitoring food consumption is of great importance in those studies aimed at evaluating the effects of bioactive compounds from plant food. Mobile phone applications for dietary intake monitoring are available in many countries $[35,36]$, and it has been reported that a 12-hour dietary recall app was in good agreement with the two reference methods (food frequency questionnaire and four dietary records) and useful for categorizing individuals according to their habitual intake of selected food and drink groups [37].

Only few of the reviewed studies included CB-product consumption among exclusion criteria and/or have instructed volunteers to avoid their consumption, whereas none of them have monitored the diet (Table 4).

We could not analyse the effects of different study designs (crossover/parallel), due to the low number of studies. As previously suggested [24], parallel design could lead to ineffective randomization and potential confounding, while the correct washout period in crossover design, in order to prevent a carry-over (or residual) effect, is difficult to establish in absence of bioavailability data, as in the reviewed studies (Table 4).

$\mathrm{CB}$ and its bioactive compounds, in vitro, inhibited enzymes and transporters involved in drug bioavailability and pharmacokinetics $[38,39]$. Although only few cases have been reported [38], probably related to genetic polymorphisms in the enzymes or transporters [40], potential fooddrug interactions should be monitored in individuals with SCI under treatment for NLUTD with drugs including oxybutynin and solifenacin [41]. The panel of experts of the "clinical guidelines for the diagnosis and treatment of lower urinary tract dysfunction in patients with SCI" concluded that anticholinergic (oxybutynin) and $\beta 3$-adrenoceptor agonist (mirabegron) drugs are recommended for patients at risk of renal damage, with symptomatic UTI or urinary incontinence [10]. However, adverse anticholinergic events can occur in SCI patients, including blurred vision, dry mouth, and constipation [10]. In this context, neurogenic bowel dysfunction (NBD), comorbidity, and polypharmacy and potential nutraceutical-drug interactions should be monitored [33], in particular in aged patients with SCI [42]. With ageing, individuals with SCI have an increased risk of renal stones [42], and the effect of CB on nephrolithiasis is controversial $[43,44]$.
Despite the large age range of many reviewed studies (Table 2), comorbidity and drug use were not reported (Table 4), and only antimicrobials and drugs acting on the immune system were among the exclusion criteria (Table 2).

Only a study evaluated the effect of CB juice (Table 3 ) and did not observe differences in bacteriuria compared to water (Table 3 and Figure 2). Reid et al. [26] reported that CB juice ingestion, but not water intake, decreased the adhesion of Gram-negative $(p=0.054)$ and Gram-positive $(p=0.022)$ bacteria from the urinary sample to uroepithelial cells, despite the similar reduction of bacteriuria compared to baseline. In addition to urine culture, the authors suggested that further parameters need to be tested in patients with SCI, since urine alone is a poor indicator of bacterial colonization [26]. Accordingly, CB products do not appear to inhibit bacterial growth or sterilize the urinary tract, and among the suggested mechanisms explaining the preventive effects of CB consumption against UTI, the hypothesis concerning acidification of urine, due to the excretion of the bacteriostatic hippuric acid, has currently been disproved [45].

On the other hand, PAC, especially A-linked PAC, had an antiadhesion effect of P-fimbriated uropathogenic Escherichia coli to uroepithelial cells, whereas B-linked PAC, found in green tea, dark chocolate, grapes, and apples, did not have this antiadhesion activity [45]. It has been reported that mucosal production of interleukin-6 (IL-6) was due to an adhesion-dependent interaction of bacteria with the mucosa [46], and it has been suggested that CB may reduce UTI symptoms through anti-inflammatory mechanisms [45]. Despite this, urinary or serum cytokines have not been included among the outcomes in the reviewed studies.

\subsection{Agreements and Disagreements with Other Reviews. The} presence of mixed treatment groups $(\mathrm{CB}$ and $\mathrm{CB}+\mathrm{MH})$ and the low risk of incomplete outcome data (Table 1) assigned by Jepson et al. [17] to the study of Lee et al. [28], which included in the analysis also individuals who had discontinued the treatment, are in our opinion a bias in evaluating the efficacy.

As reported in the meta-analysis by Jepson et al. [17] the "gold standard" bacteriological criteria for diagnosis of UTI includes bacteriuria greater than 100,000 bacterial $\mathrm{cfu} / \mathrm{mL}$ from the midstream specimen of urine, whereas for the catheter specimen of urine, a bacteriuria $<100,000 / \mathrm{mL}$ is acceptable. Despite this recommendation, only one of the reviewed studies gave a different bacteriuria threshold for midstream and catheter specimens of urine (Table 2), despite the high variability in NLUTD management, due to the differences in lesion level and completeness among patients (Table 2). Cervical SCI has distinct features compared to other SCI, caused by the impairment of manual dexterity, preventing individuals from carrying out self-catheterization (complete injury above C5 and C6), and it is often accompanied by autonomic dysfunction [10]. The latter develops in patients with high-level SCI (above T6), and bladder and bowel distension are the leading causes of autonomic dysfunction [10]. Autonomic dysfunction (above T6) is among the symptoms suggestive of UTI that can be used for the diagnosis of 
symptomatic UTI in conjunction with significant bacteriuria and pyuria $[10,12,13,47]$. In some of the reviewed studies, symptomatic UTI diagnosis required also autonomic dysfunction in individuals with lesions above T6. However, in some cases, symptomatic UTI was among the exclusion criteria (Table 2). This choice could be a bias for the evaluation of efficacy due to the low risk of UTI in the control group (Figure 3). Previous reviews did not consider these aspects, as well as bias included in the quality score described in Table 4.

\section{Conclusion}

5.1. Implication for Practice. The low quality of the reviewed studies makes it impossible to recommend or exclude the use of $\mathrm{CB}$ for preventing bacteriuria in individuals with SCI. On the other hand, it must be considered that fluid intake (2$2.5 \mathrm{~L} /$ day) is part of NBD management [33], and reductions greater than threefold of UTI incidence have been documented when NBD was reduced in individuals with SCI [47]. Therefore, in clinical practice, we suggest the evaluation of comorbidity and accurate diet and fluid intake monitoring. Previously, mobile phone applications were used for fluid and/or food intake recording and monitoring in the management of patients undergoing dialysis [48] or with diabetes [49], age-related macular degeneration [50], and cancer [51]. In addition, a risk evaluation for potential food-drug or nutraceutical-drug interactions, due to treatment for NLUTD and comorbidity, should be performed in a personalized patient-centered approach [6].

5.2. Implication for Research. A possible solution to overcome the observed variability among individuals (Table 2) could be the crossover design and monitoring of total polyphenols or of their metabolites identified in plasma and urine after the consumption of $\mathrm{CB}$ juice, including cinnamic, dihydrocinnamic, phenylacetic, benzoic, and hippuric acids; flavonols; benzaldehydes; catechols; valerolactones; and/or pyrogallols [32]. The pentacyclic triterpene ursolic acid has been suggested as the main compound in $\mathrm{CB}$ able to inhibit the activity of cyclooxygenase-2, and $\mathrm{CB}$ extracts inhibited nuclear factor $\kappa \mathrm{B}$ transcriptional activation in $\mathrm{T}$ lymphocytes and suppressed the release of IL-6, IL- $1 \beta$, IL8 , and tumor necrosis factor-alpha (TNF- $\alpha$ ) from Escherichia coli lipopolysaccharide-stimulated peripheral blood mononuclear cells [45].

Serum levels of inflammatory cytokines, including TNF$\alpha$ and IL-6, were higher in individuals with SCI, compared to healthy controls, and were further elevated in SCI subjects with UTI [52]. In a pilot study on pregnant women, CB juice consumption reduced urinary IL-6 [53], which has been suggested for differentiating between lower UTI and pyelonephritis [54].

For future research, we suggest crossover design with appropriate washout period in order to overcome variability among subjects and to consider plasma and urinary markers of inflammation as outcomes in addition to symptomatic UTI.

\author{
Abbreviations \\ ASIA: American Spinal Injury Association \\ CB: Cranberry \\ GRADE: Grading of Recommendations Assessment, \\ Development and Evaluation \\ IL: Interleukin \\ MH: Methenamine hippurate \\ NBD: Neurogenic bowel dysfunction \\ NLUTD: Neurogenic lower urinary tract dysfunction \\ NNT: $\quad$ Number needed to treat \\ PAC: Proanthocyanidins \\ RR: Risk ratio \\ SCI: $\quad$ Spinal cord injury \\ TNF: Tumor necrosis factor \\ UTI: Urinary tract infection.
}

\section{Data Availability}

The data used to support the findings of this study are available from the corresponding author upon request.

\section{Conflicts of Interest}

The authors declare no conflict of interest.

\section{Authors' Contributions}

I.P. was responsible for the conceptualization; A.R. and E.T. were responsible for the data search; A.R., E.T., and I.P. were responsible for the study selection; A.R., E.T., and I.P. were responsible for the data extraction; A.R., T.S., and I.P. were responsible for quality assessment; A.R. A.L.F., and I.P. were responsible for the formal analysis; I.P. was responsible for writing the manuscript; and T.S., A.L.F., and E.T. were responsible for the review and editing. All authors have read and agreed to the published version of the manuscript.

\section{Acknowledgments}

This research was funded by Ministero della Difesa, Italy, Project AMAMP (2019-2021).

\section{References}

[1] Asia and ISIS Committee, "The 2019 revision of the International Standards for Neurological Classification of Spinal Cord Injury (ISNCSCI)—what's new?," Spinal Cord, vol. 57, no. 10, pp. 815-817, 2019.

[2] M. Fehlings, A. Singh, L. Tetreault, S. Kalsi-Ryan, and A. Nouri, "Global prevalence and incidence of traumatic spinal cord injury," Clinical Epidemiology, vol. 6, pp. 309-331, 2014.

[3] for the Italian SCI Study Group, S. Ferro, L. Cecconi et al., "Incidence of traumatic spinal cord injury in Italy during 2013-2014: a population-based study," Spinal Cord, vol. 55, no. 12, pp. 1103-1107, 2017.

[4] J. C. Furlan, S. Gulasingam, and B. C. Craven, "Epidemiology of war-related spinal cord injury among combatants: a systematic review," Global Spine Journal, vol. 9, no. 5, pp. 545-558, 2018. 
[5] T. Sciarra, M. Ciccotti, P. Aiello et al., "Polypharmacy and nutraceuticals in veterans: pros and cons," Frontiers in Pharmacology, vol. 10, p. 994, 2019.

[6] M. Ciccotti, A. Raguzzini, T. Sciarra et al., "Nutraceuticalbased integrative medicine: adopting a Mediterranean diet pyramid for attaining healthy ageing in veterans with disabilities," Current Pharmaceutical Design, vol. 24, no. 35, pp. 4186-4196, 2019.

[7] M. H. Rabadi and C. Aston, "Evaluate the impact of neurogenic bladder in veterans with traumatic spinal cord injury," The Journal of Spinal Cord Medicine, vol. 39, no. 2, pp. 175179, 2015.

[8] F. Skelton, L. Grigoryan, S. A. Holmes, I. O. Poon, and B. Trautner, "Routine urine testing at the spinal cord injury annual evaluation leads to unnecessary antibiotic use: a pilot study and future directions," Archives of Physical Medicine and Rehabilitation, vol. 99, no. 2, pp. 219-225, 2018.

[9] M. A. Fitzpatrick, K. J. Suda, N. Safdar et al., "Changes in bacterial epidemiology and antibiotic resistance among veterans with spinal cord injury/disorder over the past 9 years," The Journal of Spinal Cord Medicine, vol. 41, no. 2, pp. 199-207, 2017.

[10] N. Sekido, Y. Igawa, H. Kakizaki et al., "Clinical guidelines for the diagnosis and treatment of lower urinary tract dysfunction in patients with spinal cord injury," International Journal of Urology, vol. 27, no. 4, pp. 276-288, 2020.

[11] J. Pannek, "Prevention of recurrent urinary tract infections in neurourology," European Urology Focus, vol. 6, no. 5, pp. 817-819, 2020.

[12] L. E. Nicolle, K. Gupta, S. F. Bradley et al., "Clinical practice guideline for the management of asymptomatic bacteriuria: 2019 update by the Infectious Diseases Society of America," Clinical Infectious Diseases, vol. 68, no. 10, pp. e83-e110, 2019.

[13] S. Compton, L. Trease, C. Cunningham, and D. Hughes, "Australian Institute of Sport and the Australian Paralympic Committee position statement: urinary tract infection in spinal cord injured athletes," British Journal of Sports Medicine, vol. 49, no. 19, pp. 1236-1240, 2015.

[14] Z. Fu, D. Liska, D. Talan, and M. Chung, "Cranberry reduces the risk of urinary tract infection recurrence in otherwise healthy women: a systematic review and meta-analysis," The Journal of Nutrition, vol. 147, no. 12, pp. 2282-2288, 2017.

[15] D. J. Liska, H. J. Kern, and K. C. Maki, "Cranberries and urinary tract infections: how can the same evidence lead to conflicting advice?," Advances in Nutrition, vol. 7, no. 3, pp. 498-506, 2016.

[16] S. Sappal, L. L. Goetz, R. Vince, and A. P. Klausner, "Randomized trial of concentrated proanthocyanidins (PAC) for acute reduction of bacteriuria in male veterans with spinal cord injury utilizing clean intermittent catheterization," Spinal Cord Series And Cases, vol. 4, no. 1, p. 58, 2018.

[17] R. G. Jepson, G. Williams, and J. C. Craig, "Cranberries for preventing urinary tract infections," Cochrane Database of Systematic Reviews, vol. 10, no. 10, article CD001321, 2012.

[18] C. H. Wang, C. C. Fang, N. C. Chen et al., "Cranberry-containing products for prevention of urinary tract infections in susceptible populations: a systematic review and meta-analysis of randomized controlled trials," Archives of Internal Medicine, vol. 172, no. 13, pp. 988-996, 2012.

[19] A. Luis, F. Domingues, and L. Pereira, "Can cranberries contribute to reduce the incidence of urinary tract infections? A systematic review with meta-analysis and trial sequential analysis of clinical trials," The Journal of Urology, vol. 198, no. 3, pp. 614-621, 2017.

[20] A. Navarrete-Opazo, P. Cuitino, and I. Salas, "Effectiveness of dietary supplements in spinal cord injury subjects," Disability and Health Journal, vol. 10, no. 2, pp. 183-197, 2017.

[21] C. Richard, C. Bendavid, J. Hascoet et al., "Urinary biomarkers profiles in patients with neurogenic detrusor overactivity according to their neurological condition," World Journal of Urology, vol. 38, no. 9, pp. 2261-2268, 2020.

[22] H. Mutlu and Z. Ekinci, "Urinary tract infection prophylaxis in children with neurogenic bladder with cranberry capsules: randomized controlled trial," International Scholarly Research Notices, vol. 2012, Article ID 317280, 4 pages, 2012.

[23] J. Kumar, M. Afsal, and A. Garg, "Imaging spectrum of spinal dysraphism on magnetic resonance: a pictorial review," World Journal of Radiology, vol. 9, no. 4, pp. 178-190, 2017.

[24] I. Peluso, A. Raguzzini, and M. Serafini, "Effect of flavonoids on circulating levels of TNF- $\alpha$ and IL- 6 in humans: a systematic review and meta-analysis," Molecular Nutrition \& Food Research, vol. 57, no. 5, pp. 784-801, 2013.

[25] T. A. Linsenmeyer, B. Harrison, A. Oakley, S. Kirshblum, J. A. Stock, and S. R. Millis, "Evaluation of cranberry supplement for reduction of urinary tract infections in individuals with neurogenic bladders secondary to spinal cord injury. A prospective, double-blinded, placebo-controlled, crossover study," The Journal of Spinal Cord Medicine, vol. 27, no. 1, pp. 29-34, 2016.

[26] G. Reid, J. Hsiehl, P. Potter et al., "Cranberry juice consumption may reduce biofilms on uroepithelial cells: pilot study in spinal cord injured patients," Spinal Cord, vol. 39, no. 1, pp. 26-30, 2001.

[27] K. B. Waites, K. C. Canupp, S. Armstrong, and M. J. DeVivo, "Effect of cranberry extract on bacteriuria and pyuria in persons with neurogenic bladder secondary to spinal cord injury," The Journal of Spinal Cord Medicine, vol. 27, no. 1, pp. 35-40, 2004.

[28] B. B. Lee, M. J. Haran, L. M. Hunt et al., "Spinal-injured neuropathic bladder antisepsis (SINBA) trial," Spinal Cord, vol. 45, no. 8, pp. 542-550, 2007.

[29] M. J. Hess, P. E. Hess, M. R. Sullivan, M. Nee, and S. V. Yalla, "Evaluation of cranberry tablets for the prevention of urinary tract infections in spinal cord injured patients with neurogenic bladder," Spinal Cord, vol. 46, no. 9, pp. 622-626, 2008.

[30] J. IntHout, J. P. Ioannidis, M. M. Rovers, and J. J. Goeman, "Plea for routinely presenting prediction intervals in metaanalysis," BMJ Open, vol. 6, no. 7, article e010247, 2016.

[31] S. A. Bahlool, "Use of L'Abbe plot in meta-analysis," British Journal of Anaesthesia, vol. 107, no. 1, p. 104, 2011.

[32] R. P. Feliciano, C. Mills, G. Istas, C. Heiss, and A. RodriguezMateos, "Absorption, metabolism and excretion of cranberry (poly)phenols in humans: a dose response study and assessment of inter-individual variability," Nutrients, vol. 9, no. 3, p. 268, 2017.

[33] M. Bernardi, A. L. Fedullo, E. Bernardi et al., "Diet in neurogenic bowel management: a viewpoint on spinal cord injury," World Journal of Gastroenterology, vol. 26, no. 20, pp. 24792497, 2020.

[34] Y. C. Chen, C. C. Chang, T. H. T. Chiu, M. N. Lin, and C. L. Lin, "The risk of urinary tract infection in vegetarians and 
non-vegetarians: a prospective study," Scientific Reports, vol. 10, no. 1, p. 906, 2020.

[35] F. Mandracchia, E. Llaurado, L. Tarro et al., "Potential use of mobile phone applications for self-monitoring and increasing daily fruit and vegetable consumption: a systematized review," Nutrients, vol. 11, no. 3, p. 686, 2019.

[36] M. R. Jospe, K. A. Fairbairn, P. Green, and T. L. Perry, "Diet app use by sports dietitians: a survey in five countries," JMIR mHealth and uHealth, vol. 3, no. 1, article e7, 2015.

[37] L. M. Bejar, O. A. Reyes, and M. D. Garcia-Perea, "Electronic 12-hour dietary recall (e-12HR): comparison of a mobile phone app for dietary intake assessment with a food frequency questionnaire and four dietary records," JMIR mHealth and uHealth, vol. 6, no. 6, article e10409, 2018.

[38] N. R. Srinivas, "Cranberry juice ingestion and clinical drugdrug interaction potentials; review of case studies and perspectives," Journal of Pharmacy \& Pharmaceutical Sciences, vol. 16, no. 2, pp. 289-303, 2013.

[39] H. Bartikova, I. Bousova, P. Jedlickova, K. Lnenickova, L. Skalova, and B. Szotakova, "Effect of standardized cranberry extract on the activity and expression of selected biotransformation enzymes in rat liver and intestine," Molecules, vol. 19, no. 9, pp. 14948-14960, 2014.

[40] M. Chen, S. Y. Zhou, E. Fabriaga, P. H. Zhang, and Q. Zhou, "Food-drug interactions precipitated by fruit juices other than grapefruit juice: an update review," Journal of Food and Drug Analysis, vol. 26, no. 2, pp. S61-S71, 2018.

[41] P. Pasko, T. Rodacki, R. Domagala-Rodacka, and D. Owczarek, "A short review of drug-food interactions of medicines treating overactive bladder syndrome," International Journal of Clinical Pharmacy, vol. 38, no. 6, pp. 13501356, 2016.

[42] L. W. Chan, T. L. Griebling, E. P. Arnold, P. S. Chu, P. W. New, and A. Wagg, "Special considerations in the urological management of the older spinal cord injury patient," World Journal of Urology, vol. 36, no. 10, pp. 1603-1611, 2018.

[43] E. J. Redmond, C. F. Murphy, J. Leonard et al., "The influence of dietary supplementation with cranberry tablets on the urinary risk factors for nephrolithiasis," World Journal of Urology, vol. 37, no. 3, pp. 561-566, 2019.

[44] T. McHarg, A. Rodgers, and K. Charlton, "Influence of cranberry juice on the urinary risk factors for calcium oxalate kidney stone formation," BJU International, vol. 92, no. 7, pp. 765-768, 2003.

[45] I. Vasileiou, A. Katsargyris, S. Theocharis, and C. Giaginis, "Current clinical status on the preventive effects of cranberry consumption against urinary tract infections," Nutrition Research, vol. 33, no. 8, pp. 595-607, 2013.

[46] P. de Man, C. van Kooten, L. Aarden, I. Engberg, H. Linder, and C. S. Eden, "Interleukin-6 induced at mucosal surfaces by gram-negative bacterial infection," Infection and Immunity, vol. 57, no. 11, pp. 3383-3388, 1989.

[47] M. Kennelly, N. Thiruchelvam, M. A. Averbeck et al., "Adult neurogenic lower urinary tract dysfunction and intermittent catheterisation in a community setting: risk factors model for urinary tract infections," Advances in Urology, vol. 2019, Article ID 2757862, 13 pages, 2019.

[48] L. C. S. Pinto, M. C. Andrade, R. O. Chaves et al., "Development and validation of an application for follow-up of patients undergoing dialysis: NefroPortátil," Journal of Renal Nutrition, vol. 30, no. 4, pp. e51-e57, 2020.
[49] J. Porter, C. E. Huggins, H. Truby, and J. Collins, "The effect of using mobile technology-based methods that record food or nutrient intake on diabetes control and nutrition outcomes: a systematic review," Nutrients, vol. 8, no. 12, p. 815, 2016.

[50] Z. C. Ali, R. Silvioli, A. Rajai, and T. M. Aslam, "Feasibility of use of a mobile application for nutrition assessment pertinent to age-related macular degeneration (MANAGER2)," Translational Vision Science \& Technology, vol. 6, no. 1, p. 4, 2017.

[51] T. Orlemann, D. Reljic, B. Zenker et al., "A novel mobile phone app (OncoFood) to record and optimize the dietary behavior of oncologic patients: pilot study," JMIR Cancer, vol. 4, no. 2, article e10703, 2018.

[52] A. L. Davies, K. C. Hayes, and G. A. Dekaban, "Clinical correlates of elevated serum concentrations of cytokines and autoantibodies in patients with spinal cord injury," Archives of Physical Medicine and Rehabilitation, vol. 88, no. 11, pp. 1384-1393, 2007.

[53] D. A. Wing, P. J. Rumney, S. Y. Leu, and F. Zaldivar, "Comparison of urinary cytokines after ingestion of cranberry juice cocktail in pregnant subjects: a pilot study," American Journal of Perinatology, vol. 27, no. 2, pp. 137-142, 2010.

[54] N. Nanda and M. Juthani-Mehta, "Novel biomarkers for the diagnosis of urinary tract infection-a systematic review," Biomarker Insights, vol. 4, 2009. 